\title{
Quantitative study of gait and balance in normal pressure hydrocephalus
}

\author{
Stephen Dombrowski*1, Brian L Davis ${ }^{2}$, Karolyn Ligon ${ }^{1}$, Judith Becker ${ }^{1}$, \\ Brandy Wozniak ${ }^{2}$ and Mark Luciano ${ }^{1}$
}

\author{
Address: ${ }^{1}$ Department of Neurosurgery, Section of Pediatric and Congenital Neurosurgery, The Cleveland Clinic, 9500 Euclid Avenue, Cleveland, \\ OH 44195, USA and 2Department of Biomedical Engineering, The Cleveland Clinic, 9500 Euclid Avenue, Cleveland, OH 44195, USA \\ Email: Stephen Dombrowski* - dombros@ccf.org \\ * Corresponding author
}

from 52nd Annual Meeting of the Society for Research into Hydrocephalus and Spina Bifida

Providence, RI, USA. II-I4 June 2008

Published: 3 February 2009

Cerebrospinal Fluid Research 2009, 6(SuppI I):S39 doi:I0.II86/I743-8454-6-SI-S39

This abstract is available from: http://www.cerebrospinalfluidresearch.com/content/6/SI/S39

(C) 2009 Dombrowski et al; licensee BioMed Central Ltd.

\section{Background}

While gait impairment and postural instability are often observed in Normal Pressure Hydrocephalus (NPH), the severity and pattern of these deficits at presentation and after treatment are uncertain. To date, qualitative clinical assessment of gait and posture has been, in large part, standard of care in the diagnosis and treatment of NPH. Recent advances in biomechanical technology are now available that can provide quantitative measures to supplement and/or extend standard clinical assessment and to further distinguish NPH from other co-morbidities. In this investigation, we demonstrate the usefulness of four newly-developed biomechanical apparati (Cranial Accelerometer, Force Plate, Kinematic Gait and Dynamic Treadmill) to quantify gait impairment and postural instability in patients being diagnosed and surgically treated for $\mathrm{NPH}$.

\section{Materials and methods}

This is a prospective clinical outcome study of sixty-two patients screened for the diagnosis of NPH through 36hour, CSF trial drainage to determine their candidacy for CSF shunt treatment which, in part, employs standard gait assessment (time* steps/m). In addition to our standard screening for functional improvement, each subject was evaluated on Cranial Accelerometer, Force Plate, Kinematic Gait and Dynamic Treadmill, before and after 36hour, continuous trial CSF lumbar drainage (CCF-NPH
Protocol) and after 3 months. Data was analyzed at presentation and after drainage, and compared between responders and non-responders.

\section{Results}

Overall, in sixty-two patients ( $32 \mathrm{M} / 30 \mathrm{~F}$ ) ranging in age from 47-94 y.o. (mean 72.6) we obtained quantitative measures in each of the four biometric tests at presentation and after extended CSF drainage. Mainly, we observed quantitative gait and postural changes after CSF trial drainage. Specifically, Cranial Accelerometer data showed significant changes in head movement (H1, H2, and CTI) corresponding to "A-P sway" which were greatly exaggerated during eyes-closed task. Using the Force Plate, we found significant changes in postural movement in both the $X$ and $Y$ planes, and Total Path Length (TPL) during eyes-open and eyes-closed tasks, which correlated with Cranial Accelerometer results in responders and nonresponders. Kinematic Gait showed improvement ( 10$20 \%$ ) in speed, stride length, step width, and turning speed which responders were significantly better than non-responders after CSF drainage. Dynamic Treadmill data revealed significant differences between responders and non-responders in symmetry for left/right vertical forces and medial-lateral forces after CSF drainage. In responders, improvement in gait and posture observed with trial CSF drainage was often, but not always retained at $3 \mathrm{M}$ post-shunt treatment; by comparison, non- 
responders performance showed no change or deterioration at $3 \mathrm{M}$ follow up. Several quantitative gait and postural measures significantly correlated with standard gait assessment.

\section{Conclusion}

This study provides accurate and reliable quantitative data that reflects the gait and postural impairment observed clinically in patients with NPH. Importantly, we were able to confirm and extend gait and postural improvement in patients undergoing CSF trial drainage using the Cranial Accelerometer, Force Plate, Kinematic Gait and Dynamic Treadmill testing which is currently being assessed clinically. At this time, quantitative gait and postural evaluation may not supplant but assist in clinical diagnosis and treatment of NPH.

\section{Acknowledgements}

Support for this investigation provided by Medtronic, Inc. and Codman \&

Shurtleff, Inc., a Johnson \& Johnson Co.
Publish with Biomed Central and every scientist can read your work free of charge

"BioMed Central will be the most significant development for disseminating the results of biomedical research in our lifetime. " Sir Paul Nurse, Cancer Research UK

Your research papers will be:

- available free of charge to the entire biomedical community

- peer reviewed and published immediately upon acceptance

- cited in PubMed and archived on PubMed Central

- yours - you keep the copyright

Submit your manuscript here:

http://www.biomedcentral.com/info/publishing_adv.asp 